\title{
LncRNA H19 promotes the committed differentiation of stem cells from apical papilla via miR-141/SPAG9 pathway
}

Zehan Li ${ }^{1,2}$, Ming Yan ${ }^{1,2}$, Yan Yu ${ }^{1,2}$, Yanqiu Wang ${ }^{1,2}$, Gang Lei ${ }^{1,2}$, Yin Pan ${ }^{1,2}$, Na Li $^{1,2}$, Romila Gobin ${ }^{1}$ and Jinhua Yu (D)

\begin{abstract}
Long noncoding RNAs (IncRNAs) exert significant roles at transcriptional and post-transcriptional levels. Stem cells from apical papilla (SCAPs) differentiate into dentin/bone-like tissues under certain conditions. So far, whether IncRNAH19 can affect the proliferative behaviors and osteo/odontogenesis of SCAPs, as well as its specific mechanism remain to be elucidated. Here, SCAPs were isolated and transfected with the lentiviruses or packaging vectors. Our results showed that InCRNA-H19 had no significant effect on the proliferative behaviors of SCAPs, as presented by CCK-8 assay, EdU assay and flow cytometry (FCM). Furthermore, alkaline phosphatase (ALP) activity, alizarin red staining, Western blot assay (WB), quantitative real-time polymerase chain reaction (qRT-PCR) and in vivo bone formation assay were conducted to verify the biological influences of H19 on SCAPs. Overexpression of H19 led to the enhanced osteo/odontogenesis of SCAPs, whereas knockdown of H19 inhibited these effects. Mechanistically, H19 competitively bound to miR-141 and prevented SPAG9 from miRNA-mediated degradation, thus significantly elevating phosphorylated levels of p38 and JNK and facilitating the committed differentiation of SCAPs. Taken together, the osteo/odontogenesis of SCAPs was upregulated by overexpression of H19 via miR-141/SPAG9 pathway.
\end{abstract}

\section{Introduction}

Regeneration of the lost bone is very important in diseases with bone loss, such as tumors, bony defects and fractures. In recent years, mesenchymal stem cells (MSCs)-based cellular therapies present a promising prospect for bone defect treatment ${ }^{1}$. MSCs could differentiate into a variety of adult cell types including osteoblasts. Due to their strong multi-potentiality and regenerative properties, biological functions of MSCs have been well recognized and their researches on bone tissue engineering achieved great process ${ }^{2,3}$. Moreover, they can be isolated from numerous tissues in humans, such as

\footnotetext{
Correspondence: Jinhua Yu (yujinhua@njmu.edu.cn)

${ }^{1}$ Key Laboratory of Oral Diseases of Jiangsu Province and Stomatological Institute of Nanjing Medical University, 140 Hanzhong Road, Nanjing, Jiangsu 210029, China

${ }^{2}$ Endodontic Department, School of Stomatology, Nanjing Medical University, 136 Hanzhong Road, Nanjing, Jiangsu 210029, China

These authors contributed equally: Zehan Li, Ming Yan, Yan Yu

Edited by $\mathrm{G}$. Calin
}

peripheral blood, bone marrow, umbilical cord blood, placenta, and dental tissues ${ }^{4}$. However, in comparison with other sources, MSCs derived from dental tissues exist in the human body during the whole life. In addition, it is generally believed that they are extremely accessible. The isolation of MSCs from dental tissues is convenient during the procedures. Hence, they are considered to be potent candidates for bone tissue engineering ${ }^{5,6}$.

As a major kind of dental stem cells, stem cells from apical papilla (SCAPs) are essential for the developing alveolar bone, tooth root and dental pulp-dentin complex. They are isolated from the soft tissues at the apices of developing permanent teeth ${ }^{7}$. SCAPs exert advantages of self-renewing and multilineage differentiation such as osteogenic, odontogenic, adipogenic, and neurogenic ${ }^{8}$. It has been reported that SCAPs present remarkable tissue regenerative capability in spinal cord injuries ${ }^{9}$. Besides, a relative study conducted using the swine model showed the biological function tooth root produced by SCAPs ${ }^{10}$.

\section{(c) The Author(s) 2019}

(c) (i) Open Access This article is licensed under a Creative Commons Attribution 4.0 International License, which permits use, sharing, adaptation, distribution and reproduction c. in any medium or format, as long as you give appropriate credit to the original author(s) and the source, provide a link to the Creative Commons license, and indicate if changes were made. The images or other third party material in this article are included in the article's Creative Commons license, unless indicated otherwise in a credit line to the material. If material is not included in the article's Creative Commons license and your intended use is not permitted by statutory regulation or exceeds the permitted use, you will need to obtain permission directly from the copyright holder. To view a copy of this license, visit http://creativecommons.org/licenses/by/4.0/. 
Complex molecular mechanisms including signaling pathways and microRNAs underlying SCAPs osteo/ odontogenic differentiation have been extensively investigated $^{11,12}$. Our previous studies have demonstrated that many factors including growth factors (e.g., insulin-like growth factor I, IGF-I) ${ }^{13}$, bioactive materials (e.g., mineral trioxide aggregate $)^{14}$, and hormones (e.g., 17beta-estradiol) ${ }^{15}$ can affect the osteo/odontogenic differentiation of SCAPs.

In the past decades, long noncoding RNAs (lncRNAs) have exerted their biological functions in the transcriptional and post-transcriptional regulation of diverse biological processes, such as cellular progression and differentiation ${ }^{16,17}$. Recently, lncRNA expression profiles analyzed by the high throughput technologies characterized a number of osteogenesis-related lncRNAs. For example, lncRNA-TUG1 accelerates osteogenic differentiation in periodontal ligament stem cells ${ }^{18}$. LncRNAMEG3 stimulates osteogenic differentiation of MSCs as well ${ }^{19}$. LncRNA-ANCR inhibits osteogenesis through physical interaction of EZH2 and direct regulation of Run $22^{20}$. Recent studies have demonstrated that lncRNAs could serve as competing endogenous RNA (ceRNA) by interacting with the miRNA, thus regulating target gene expression $^{21,22}$.

As we all know, microRNAs (miRNAs) are major players in gene regulation through binding to the $3^{\prime}$ untranslated region ( $3^{\prime} \mathrm{UTR}$ ) of the target mRNAs, and subsequently cause mRNA degradation or translation inhibition ${ }^{23}$. LncRNA serves as a miRNA spong and relieves inhibitory effect of miRNA on target genes. For example, lncRNA-1604 sponges to miR-200c, leading to ZEB overexpression and thus promotes embryonic stem cells differentiation ${ }^{24}$. LncRNA TUG1 regulates the expression of its target FGF1 by sponging miR-133a ${ }^{25}$. LncRNA-H19 is of great significance in promoting skeletal muscle differentiation as one of the most conserved noncoding transcripts in mammalian development ${ }^{26}$. Despite the previous achievements, the specific mechanism of H19 in influencing osteo/odontogenic differentiation of SCAPs remains unknown. Here, we demonstrated for the first time that H19 promoted the osteo/odontogenic differentiation of SCAPs while miR-141 inhibited. Moreover, H19 sponged miR-141 and released its inhibitory effect on SPAG9. Our results provide references for further analysis of the IncRNA-miRNA-mRNA network during the regeneration of the bone/dentin tissues.

\section{Materials and methods Cell culture}

This study got approval of the Ethical Committee of the Stomatological School of Nanjing Medical University. Experimental procedures were conducted in accordance with the Human Care Guidelines of the Ethical
Committee of Nanjing Medical University. Impacted third molars were collected from 9 healthy donors aged 17-20 years after the informed consent was obtained in the Oral Surgery Department of Jiangsu Provincial Stomatological Hospital. The apical papilla were carefully isolated from the immature roots, cut and digested in medium containing $3 \mathrm{mg} / \mathrm{ml}$ collagenase type I and $4 \mathrm{mg} / \mathrm{ml}$ dispase (Sigma, St. Louis, MO, USA) at $37^{\circ} \mathrm{C}$. Thirty minutes later, cells were purified using rabbit anti-STRO-1 antibody (Santa Cruz, Delaware, CA) and sheep anti-rabbit IgG Dynabeads (Dynal Biotech, Oslo, Norway) followed by magnetic activated cell sorting (MACS) instructions. Isolated cells were maintained in alpha minimum essential medium ( $\alpha$-MEM, Gibco, Life Technologies, Grand Island, NY, USA) with $10 \%$ fetal bovine serum (FBS, Hyclone, Logan, UT, USA), $100 \mathrm{U} / \mathrm{mL}$ penicillin and 100 $\mathrm{mg} / \mathrm{mL}$ streptomycin at $37^{\circ} \mathrm{C}$ in a $5 \% \mathrm{CO}_{2}$ incubator. Culture medium was replaced every other day. Based on the cell surface makers, SCAPs were identified by flow cytometry (FCM) as previously described ${ }^{15}$. Third-passage cells were harvested for the subsequent experiments.

\section{Lentivirus infection}

Recombinant lentiviruses containing full-length H19 (Gene Bank accession number, NR_002196.1) and scramble control (NC) were obtained from GenePharma Company (Shanghai, China). Recombinant lentiviruses targeting H19 (Lenti-shH19-1 and Lenti-shH19-2) and scramble control (Lenti-shNC) were also obtained from GenePharma Company. SCAPs were transfected by lentiviruses exposure in $1 \mathrm{~mL}$-MEM supplemented with $10 \% \mathrm{FBS}$ and $8 \mu \mathrm{g} / \mathrm{mL}$ polybrene (POL) for $10 \mathrm{~h}$. Infected cells were cultured in the conventional medium and the expression level of $\mathrm{H} 19$ was detected.

\section{Plasmid construction and transfection of miRNA mimics/ inhibitors}

MiRNA plasmids were obtained from Ribobio Company (Guangzhou, China). SCAPs were transfected by using transfection reagent riboFECT ${ }^{\mathrm{TM}} \mathrm{CP}$ (Ribobio, Guangzhou, China). The mutated binding sites of miR-141 in luciferase reporter vectors containing H19 and SPAG9 were constructed by site-directed mutagenesis. Transient transfection was conducted using Lipofectamine 2000 (Invitrogen, USA).

\section{Flow cytometry}

Transfected cells were collected by using trypsin (Beyotime, Haimen, China) and fixed with pre-cold alcohol at $4{ }^{\circ} \mathrm{C}$ overnight in dark. After phosphate buffered saline (PBS) wash, cell cycle phases (G0/G1, S, and G2/M phases) were evaluated using FACScan flow cytometer (BD Biosciences, San Jose, CA). The experiment was repeated three times. 


\section{Cell proliferation assay}

Regulatory effects of $\mathrm{H} 19$ on proliferative potential were determined by the Cell Counting Kit-8 (CCK- 8 kit) (Dojindo, Tokyo, Japan) assay and EdU incorporation assay. Briefly, transfected SCAPs were plated into a 96well plate with $3 \times 10^{3}$ cells/well. $10 \mu \mathrm{L}$ CCK-8 reagent was added at different time points (day $0,1,3,5,7,9$, respectively), and the absorbance at $450 \mathrm{~nm}$ was measured $2 \mathrm{~h}$ later by a microplate reader. The experiment was repeated in triplicate.

For EdU incorporation assay, transfected SCAPs $(5 \times$ $10^{3}$ cells per well) received 2-h incubation with $25 \mathrm{mM} 5$ ethynyl-20-deoxyuridine (EdU, Ribobio). After fixation in $4 \%$ paraformaldehyde (PFA) for $15 \mathrm{~min}$ and induction with $0.5 \%$ Triton $\mathrm{X}-100$ for $20 \mathrm{~min}$ at room temperature, cells were treated with $1 \times$ Apollo reaction cocktail for 30 min. Subsequently, the DNA was stained with Hoechst 33342 for $20 \mathrm{~min}$ and visualized using a fluorescence microscope.

\section{Alkaline phosphatase (ALP) activity and staining}

ALP activity was recorded at $405 \mathrm{~nm}$ using an ALP activity assay kit (Jiancheng, Nanjing, China) as previously described $^{14}$. Total protein content of each sample was determined with a BCA kit (Beyotime, China). ALP activity relative to the control group was normalized to the total protein content.

According to the protocol of the NBT/BCIP staining kit (Beyotime, China, transfected SCAPs were washed with PBS and fixed in 4\% PFA for $30 \mathrm{~min}$. After PBS wash for three times, cells were incubated in alkaline solution for $20 \mathrm{~min}$ at $37^{\circ} \mathrm{C}$.

\section{Alizarin red staining and quantification}

As described previously, alizarin red staining was performed to evaluate mineralization ${ }^{13}$. Transfected SCAPs were fixed in ice-cold $70 \%$ ethyl alcohol for $30 \mathrm{~min}$ and stained with $40 \mathrm{mM} / \mathrm{L}$ alizarin red $(\mathrm{pH}=4.2$, SigmaAldrich) for $20 \mathrm{~min}$ at room temperature. Alizarin red dissolved in $10 \mathrm{mmol} / \mathrm{L}$ sodium phosphate containing $10 \%$ cetylpyridinium chloride (CPC, Sigma-Aldrich) for $30 \mathrm{~min}$ at $25^{\circ} \mathrm{C}$ was used to quantification by the spectrophotometric absorbance at $570 \mathrm{~nm}$. The final calcium concentration was normalized to the total protein content.

Real-time reverse transcription polymerase chain reaction

We used TRIzol reagent (Invitrogen, New York, NY, USA) to extract cellular RNA. RNA was determined at 230, 260, and $280 \mathrm{~nm}$, respectively. The mRNA was reversely transcribed into cDNA using the PrimeScript RT Master Mix kit (TaKaRa Biotechnology, China). RTPCR was performed using SYBR Green Master (Roche, Indianapolis, IN, USA) and ABI 7300 real-time PCR
Table 1 Sense and antisense primers for real-time reverse transcription polymerase chain reaction

\begin{tabular}{lll}
\hline Genes & Primers & Sequences (5'-3') \\
\hline DMP1 & Forward & CCCTTGGAGAGCAGTGAGTC \\
& Reverse & CTCCTTITCCTGTGCTCCTG \\
COL-1 & Forward & CCCTTTCTGCTCCTTTCT \\
& Reverse & TGTTCCTGTGTCTTCTGG \\
RUNX2 & Forward & TCTTAGAACAAATTCTGCCCTT \\
& Reverse & TGCTTGGTCTTGAAATCACA \\
DSPP & Forward & ATATTGAGGGCTGGAATGGGGA \\
& Reverse & TTTGGGCTCCAGCATTGTCA \\
OSX & Forward & CCTCCTCAGCTCACCTTCTC \\
& Reverse & GTTGGAGCCCAAATAGAAA \\
OCN & Forward & AGCAAAGGTCAGCCTTTG \\
& Reverse & GCGCCTGGGTCTCTCACT \\
H19 & Forward & CTTTCATGTTGTGGTTCTGG \\
& Reverse & CGGGTCTGTTTCTTACTTCC \\
& Forward & GGCGGCTCGAGAAATCCGTTCTA \\
& Reverse & AATGCGGCCGCAACTCAATCAAC \\
\hline
\end{tabular}

system. Primers used in this experiment were listed in Table 1. Human U6 RNA was applied as an internal control. Human GAPDH was used as a control for normalizing expressions of osteo/odontoblast-associated genes ( $A L P$, DSPP, DMP1, RUNX2, OSX, and OCN) calculated by the $2^{-\Delta \Delta \mathrm{Ct}}$ method as previously reported ${ }^{12}$.

\section{Western blot}

Cell lysates were harvested by RIPA buffer (Beyotime, China) with Complete Protease Inhibitor Cocktail (Roche, USA). Protein samples were separated by $10 \%$ SDS-PAGE and transferred to PVDF membrane (Millipore, USA). The membranes were blocked in 5\% BSA for $2 \mathrm{~h}$ at room temperature and incubated overnight with primary antibodies [OCN (ab93876, Abcam, UK), OSX (ab22552, Abcam, UK), RUNX2 (ab76956, Abcam, UK), ALP (ab95462, Abcam, UK), DSP (sc-33586, Santa Cruz), ERK (\#4695, Cell Signaling Technology), p-ERK (\#4370, Cell Signaling Technology), p38 (\#8690, Cell Signaling Technology), p-p38 (\#4511, Cell Signaling Technology), JNK (\#9252, Cell Signaling Technology), p-JNK (\#9255, Cell Signaling Technology), SPAG9 (\#5519, Cell Signaling Technology) and GAPDH (\#2118, Cell Signaling Technology)]. Membranes were washed with Tris-buffer saline containing $0.05 \%$ Tween 20 (TBST) for three times and 5 min each. The membranes were incubated with secondary antibodies for $1 \mathrm{~h}$ in room temperature, followed by TBST 
wash for $30 \mathrm{~min}$. Western blot analysis were quantified using ImageJ software (http://rsb.info.nih.gov/ij/).

\section{Immunofluorescence staining}

Transfected SCAPs were seeded onto $10 \mathrm{~mm}^{2}$ glass coverslips and cultured for three days. Cells were washed with $0.01 \mathrm{~mol} / \mathrm{L}$ PBS and fixed with $4 \%$ PFA for $30 \mathrm{~min}$ at room temperature. After PBS wash, cells were permeabilized with $0.25 \%$ Triton-100 for $12 \mathrm{~min}$, and blocked with normal goat serum (DCS/BioGenex, Hamburg, Germany) for $45 \mathrm{~min}$ at $37^{\circ} \mathrm{C}$. Incubation of primary antibodies [RUNX2 (ab76956, Abcam, UK), OSX (ab22552, Abcam, UK) and DMP1 (NBP1-45525, Novus)] were conducted for $12 \mathrm{~h}$ at $4{ }^{\circ} \mathrm{C}$, followed by secondary antibody labeling with fluorochrome for another $30 \mathrm{~min}$ at $37^{\circ} \mathrm{C}$ in dark. Coverslips were observed under the inverted fluorescence microscope (Olympus, Japan).

\section{Dual-luciferase reporter assay}

The HEK293T cells were seeded into a 24-well plate with $5 \times 10^{5}$ per well. Cells were co-transfected with luciferase plasmids and miR-141 mimic or negative control. Luciferase activities of Renilla and Firefly were measured $48 \mathrm{~h}$ after transfection using Dual Luciferase Reporter Assay System (Promega).

\section{RNA immunoprecipitation (RIP)}

Magna RIPTM RNA-Binding Protein Immunoprecipitation Kit (Millipore, Bedford, MA, USA) was used for RIP assay. Briefly, lysed cells were incubated with RIP buffer containing magnetic beads conjugated with antibodies against JIP-4 (\#5519, Cell Signaling Technology) and rabbit IgG control to precipitate the potential substances in the RISC complex. RNA purification was performed using RNase-free DNase I and proteinase $\mathrm{K}$ (Thermo Fisher Scientific, Waltham, MA, USA). The mRNA level of miR-141 in extract was detected by qRT-PCR.

\section{Bone formation assay and micro-CT analysis}

SCAPs with H19 overexpression and controls induced under osteogenic medium for $1 \mathrm{w}$ were harvested for the in vivo study. SCAPs cells incubated with Bio-Oss Collagen (Geistlich, Germany) scaffolds for $1 \mathrm{~h}$ at $37^{\circ} \mathrm{C}$ were implanted into the dorsal sides of BALB/c homozygous nude mice ( 5 weeks old, five mice in each group). Eight weeks later, implants were harvested and fixed in 4\% PFA. Animal procedures got approval by the Animal Care and Use Committee of Nanjing Medical University. Micro-CT analysis was performed using a highresolution Inveon Micro-CT (Siemens, Munich, Germany) set as $80 \mathrm{kV}$ of $\mathrm{X}$-ray source, $500 \mu \mathrm{A}$ of a node current and $500 \mathrm{~ms}$ of the 360 rotational steps per time. Micro-CT image analysis software (Inveon Research Workplace) was utilized for reconstructing image slices. The ratio of new bone volume to existing tissue volume (BV/TV) was calculated.

\section{Histological and histomorphometric analyses}

Tissues were decalcified in $10 \%$ ethylene diamine tetraacetic acid ( $\mathrm{pH} 7.4)$ for $4 \mathrm{w}$, dehydrated and paraffin embedded. Tissues were sectioned and stained with hematoxylin and eosin (H\&E) or Masson's trichrome. Ten randomly selected fields in each section were captured under the microscope. Immunohistochemical staining for OCN was performed. Decalcified sections were blocked with goat serum, incubated with primary antibodies against OCN (1:300 dilution) at $4{ }^{\circ} \mathrm{C}$ overnight, and analyzed with the $\mathrm{ABC}$ detection kit (Maixin Biotech). Immunohistochemical staining was captured under the microscope.

\section{Statistical processing}

Data analyzed with were GraphPad Prism software expressed as means $\pm \mathrm{SD}$ from three independent experiments. Statistical Package for Social Sciences (SPSS) software (version 16.0) was utilized for statistical analyses. Differences between two groups were compared using the Student's $t$ test. $P<0.05$ indicated statistically significant.

\section{Results}

LncRNA-H19 expression increases with the prolongation of osteogenic differentiation of SCAPs

To identify the underlying effect of IncRNA-H19 on the osteogenic differentiation of SCAPs, RNA samples were collected for detecting expression changes of H19. RTPCR results revealed that $H 19$ expression at day 3 and 7 of osteogenic differentiation of SCAPs was gradually upregulated $(P<0.05$, Fig. 1a). We also examined the mRNA expressions of $A L P$ and RUNX2 (the early-stage osteogenic markers), which displayed a significant increase in the osteogenic differentiation of SCAPs. Meanwhile, OCN (the late-stage marker) was also significantly upregulated with time $(P<0.05$ or $P<0.01$, Fig. 1a).

\section{LncRNA-H19 could not affect SCAPs proliferation}

To elucidate the function of lncRNA-H19 in SCAPs proliferation, lentivirus was transfected to alter H19 expression in SCAPs. Stably transfected cells were assigned into NC, H19, shNC, shH19-1, and shH19-2 groups. Transfection efficacy of $\mathrm{H} 19$ was confirmed by quantitative RT-PCR analysis $(P<0.01$, Supplemental Figs. $1 \mathrm{~A}$ and $1 \mathrm{~B})$ CCK-8 assay did not show significant difference in proliferative rate between $\mathrm{H} 19$ group and NC group or among shNC, shH19-1 and shH19-2 groups at day $9(P>0.05$, Fig. 1b). FCM analysis did not reveal distinct difference in the proliferative index $(\mathrm{PI}=\mathrm{G} 2 \mathrm{M} \pm$ S) between NC group (5.61\%) and H19 group (5.73\%, 
A

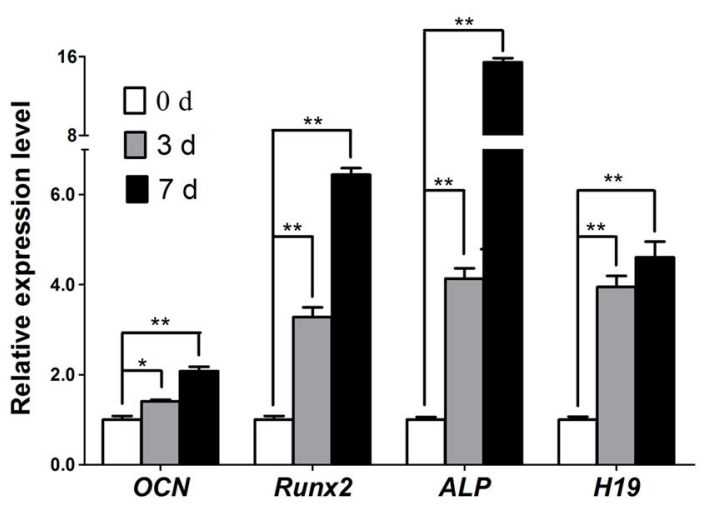

B
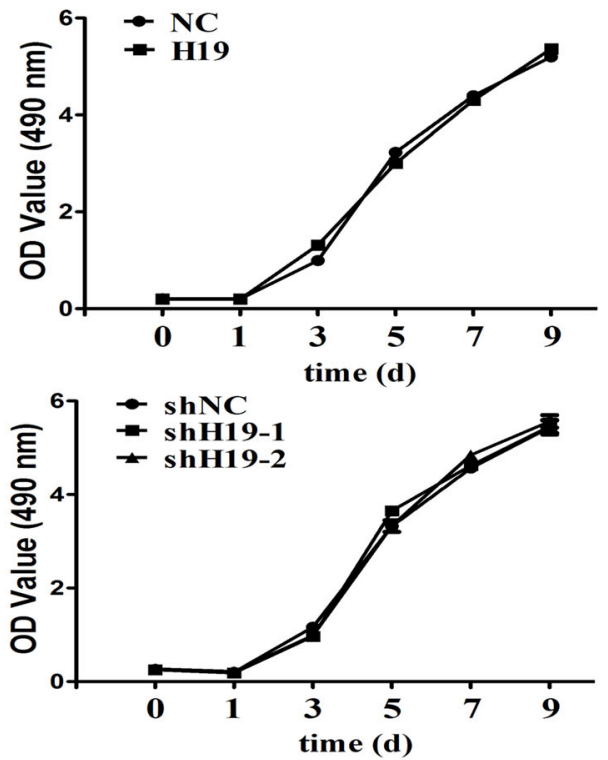

C

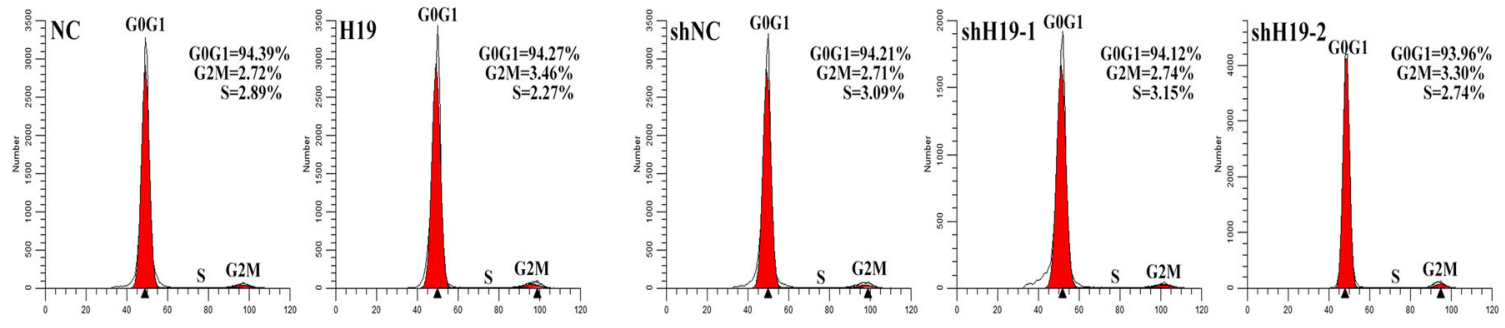

D
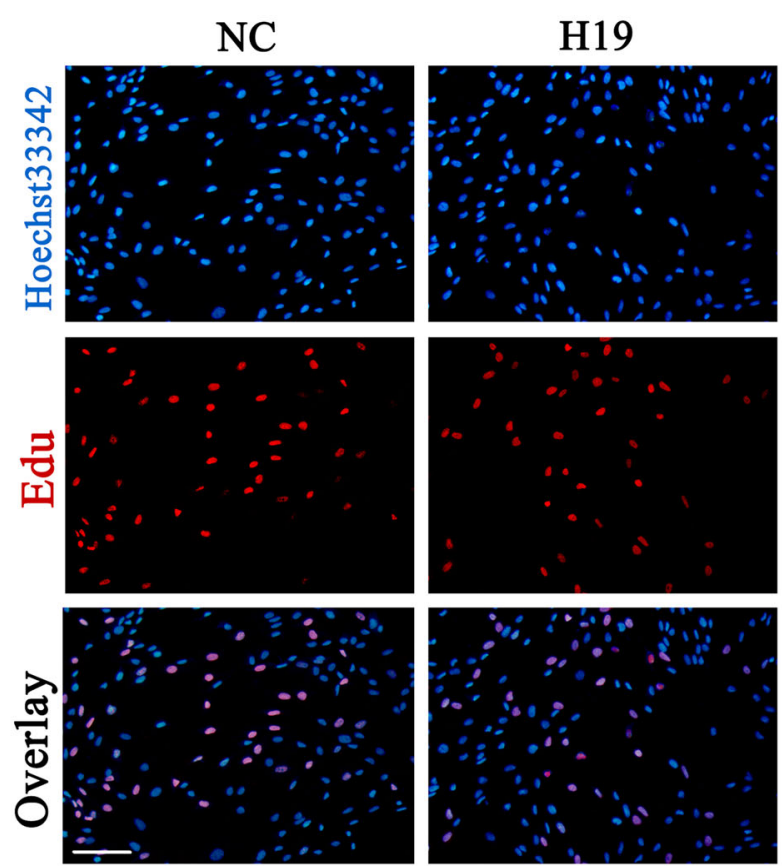

E
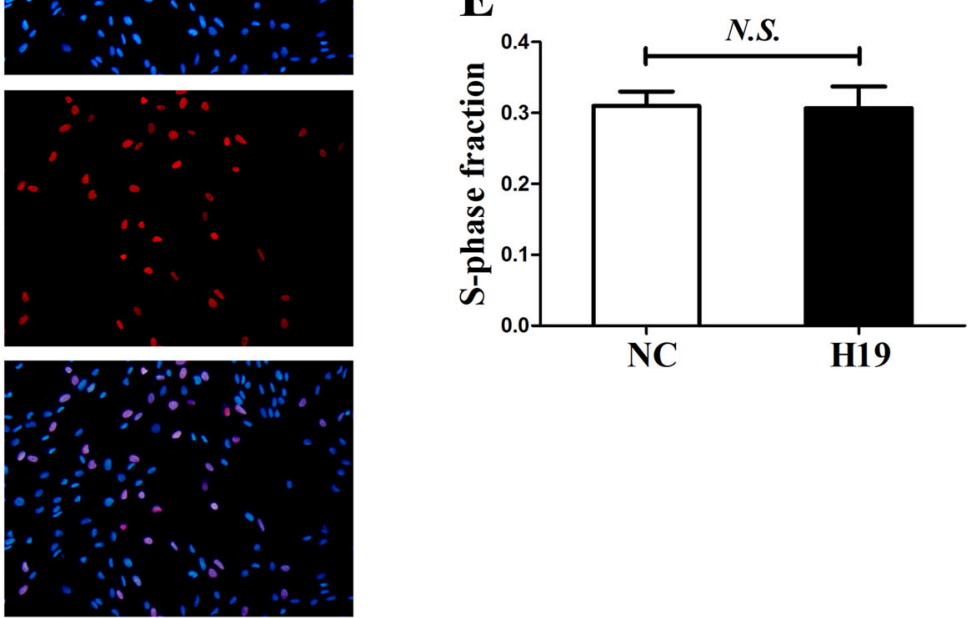

Fig. 1 (See legend on next page.) 
(see figure on previous page)

Fig. 1 LncRNA-H19 expression during osteoblast differentiation of stem cells from apical papilla (SCAPs) and effects of IncRNA-H19 on proliferation of SCAPs. a Relative expression of $\mathrm{H} 19$ and osteoblastic markers of OCN, Runx2, and ALP were determined by qRT-PCR analysis during osteoblast differentiation of SCAPs at day 0,3 and 7 . The relative expression leves at indicated time points were normalized to day 0 . GAPDH was used as an internal control. Results were presented as the mean \pm SD $\left({ }^{*} P<0.05,{ }^{*} P<0.01\right)$. b CCK-8 assay showed no significant difference in cell proliferation when $\mathrm{H} 19$ was overexpression or knockdown from day 0 to day 9. $\mathbf{c}$, d Flow cytometry and EdU assay demonstrated that $\mathrm{H} 19$ had no significant difference on cell proliferation of SCAPs at day 7 (Scale Bar $=200 \mu$ m; N.S., $P>0.05$ )

$P>0.05$, Fig. 1c). Likewise, no distinct difference was found in the proliferative index among shNC group (5.8\%), shH19-1 group (5.89\%) and shH19-2 group (6.04\%, $P>0.05$, Fig. 1c). Moreover, EdU retention assay showed no obvious difference between NC group and H19 group as well $(P>0.05$, Fig. 1e). Taken together, the data accumulated here elucidated that lncRNA-H19 could not affect SCAPs proliferation.

\section{LncRNA-H19 promotes the osteo/odontogenesis of SCAPs}

Transfected SCAPs were induced in osteoblast differentiation medium, and Western blot results demonstrated that protein expressions of OCN, OSX, RUNX2, ALP, and DSP in Lenti-H19 infected SCAPs were markedly higher than those in control group at day $7(P<0.05$ or $P<0.01$, Fig. 2a). Meanwhile, the mRNA levels of OCN, OSX, RUNX2, ALP, and DSPP also increased by H19 overexpression, whereas H19 knockdown obtained the opposite effects $(P<0.05$ or $P<0.01$, Fig. 2a, b). ALP activity was obviously upregulated by $\mathrm{H} 19$ overexpression at day 7 and H19 knockdown resulted in decreased ALP activity (Fig. 2c). After 14 days of osteogenic induction, ALP activity and matrix mineralization were markedly enhanced (Fig. 2c, d). ALP activity and matrix mineralization were even enhanced in H19 group without osteogenic supplements as compared with controls (Fig. 2c, d). CPC results also revealed that the calcium concentration in $\mathrm{H} 19$ overexpressing cells was much higher than those cells with H19 knockdown at day 14 ( $P$ $<0.01$, Fig. 2e). In addition, immunofluorescence staining showed upregulated protein levels of OSX and DSP in Lenti-H19 infected SCAPs as compared with NC group at day 7 (Fig. 2f). These results indicated that $\mathrm{H} 19$ promotes the osteo/odontogenic differentiation of SCAPs.

SCAPs stably expressing $\mathrm{H} 19$ and controls were loaded on Bio-Oss Collagen scaffolds, and implanted in the subcutaneous tissues of nude mice (five mice in each group) for 8-week growth (Fig. 3a). BV/TV increased in the H19-overexpressing group compared with the control group (Fig. 3b). Histological examination corroborated the result of BV/TV. Both $\mathrm{H} \& \mathrm{E}$ and Masson staining showed more bone-like structures and collagen deposit in SCAPs of the H19-overexpressing group than the control group (Fig. 3c). Moreover, the abundance of $\mathrm{OCN}$ increased in the H19-overexpressing group than control group (Fig. 3c).

\section{LncRNA-H19 serves as a miRNA sponge for miR-141}

To determine how $\mathrm{H} 19$ regulates the osteo/odontogenic differentiation of SCAPs, the candidate miRNAs targeting H19 was searched by miRDB and Target Scan software. MiR-141 was predicted as the potential target of lncRNAH19 (Fig. 4a). Transfection efficacy of miR-141 was verified by qRT-PCR (Supplemental Fig. 1C). H19 expression was negatively regulated by miR-141 $(P<0.01$, Fig. $4 b)$. To further determine whether H19 directly regulates miR-141, we cloned the putative miR-141 target binding sequence into a luciferase construct. MiR-141 mimics and H19 wildtype or mutant reporter were transferred into 293T cells. Luciferase activity of $\mathrm{H} 19$ wild-type reporter was markedly reduced by miR-141 mimics, and mutation of the putative miR-141 target sites successfully reversed the previous suppressive effect $(P<0.05$, Fig. $4 \mathrm{c})$. These findings suggested the binding condition between miR-141 and H19.

\section{MiR-141 inhibits the osteo/odontogenic differentiation of SCAPs}

To investigate the effect of miR-141 on the osteo/ odontogenic differentiation of SCAPs, miR-141 mimics and inhibitor were used to transiently transfect SCAPs. MiR-141 inhibited the osteo/odontogenic differentiation of SCAPs while miR-141 inhibitor increased it at day $7(P<$ 0.05 , Fig. $5 \mathrm{a}-\mathrm{C})$. Thereafter, mRNA levels of several osteo/ odontogenic marker genes were determined by qRT-PCR. MiR-141 inhibited mRNA levels of majority osteo/odontogenic marker genes $(P<0.05$ or $P<0.01$, Fig. 5 d, e). In addition, after the osteogenic induction for 14 days, overexpression of miR-141 led to a decreased calcified nodules assessed by alizarin red staining (Fig. $5 \mathrm{f}$ ), and CPC results further confirmed these results $(P<0.05$, Fig. 5 g). Moreover, ALP analysis also confirmed that miR-141 obviously alleviated the expression of ALP in the committed differentiation of SCAPs $(P<0.01$, Fig. $5 \mathrm{~h}, \mathrm{i})$. Immunofluorescence staining revealed that the protein levels of OSX and DSP remarkably decreased in SCAPs transfected with miR-141 mimics than NC group after osteogenic induction for 7 days (Fig. 5j). Collectively, the above findings proved that miRNA-141 was a negative regulator of SCAPs during the osteo/odontogenic differentiation.

\section{MiR-141 down-regulates SPAG9 expression in SCAPs}

Previous study has revealed that miR-141 could markedly inhibit SPAG9 expression $^{23}$. Furthermore, SPAG9 

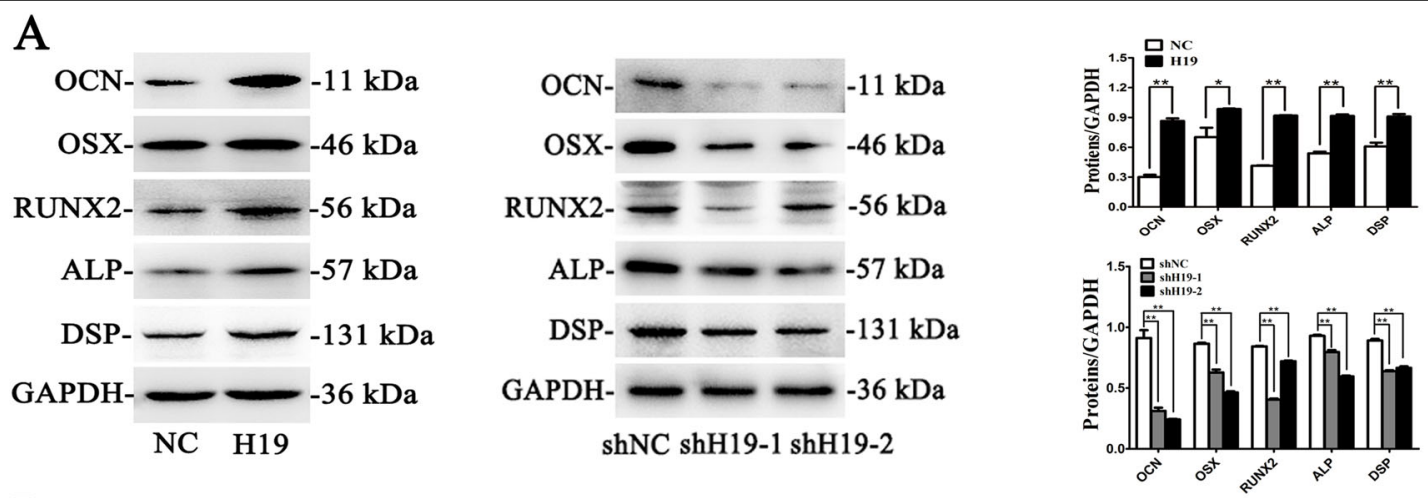

B
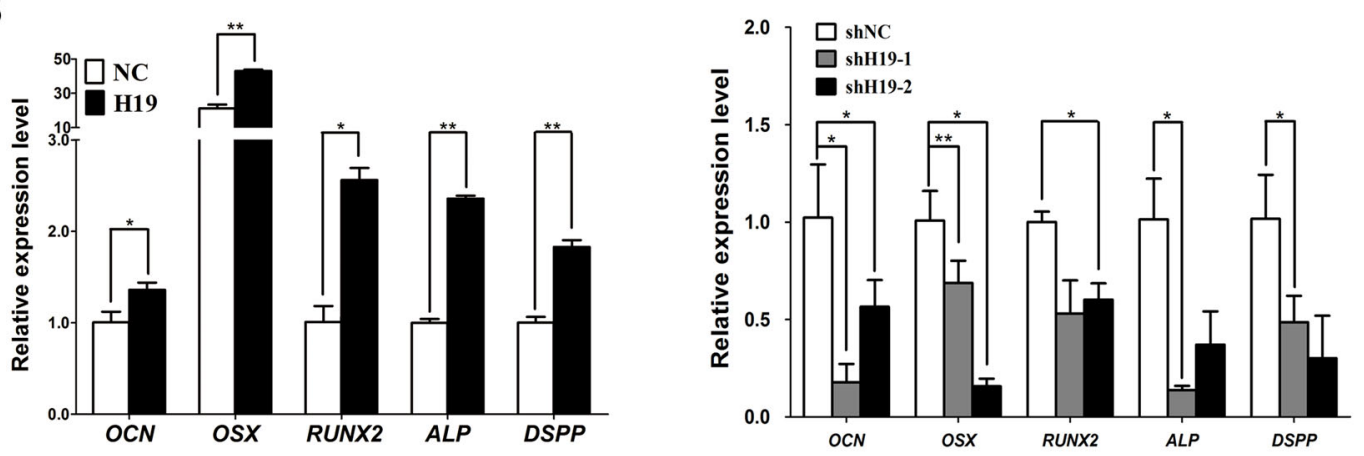

C
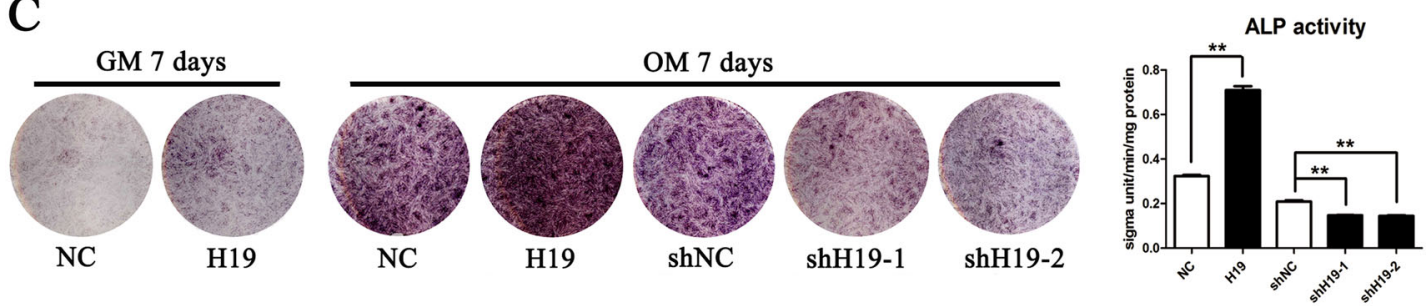

D

GM 14 days

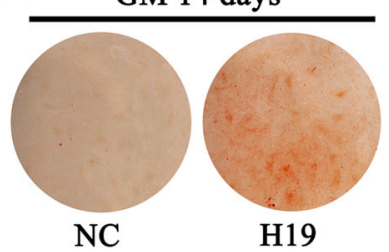

E
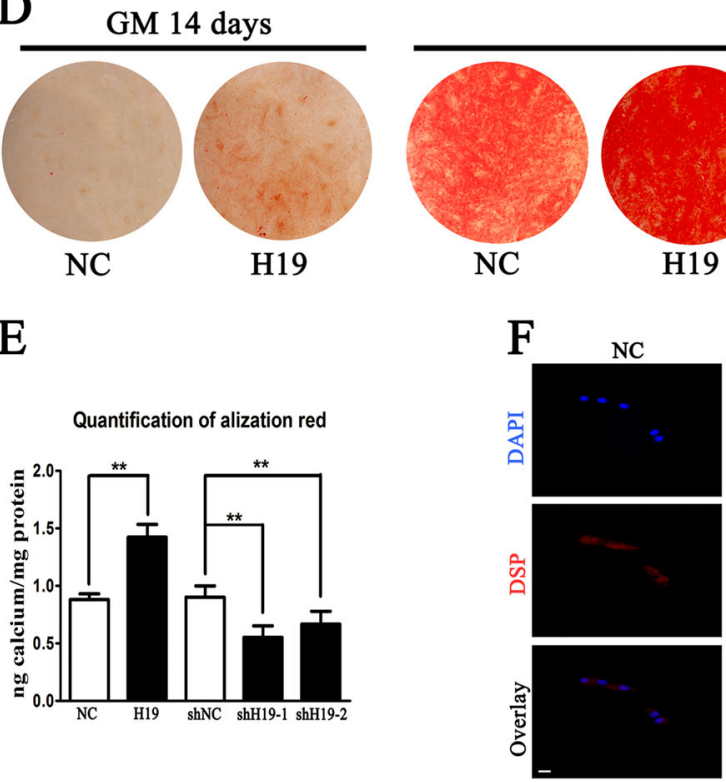

OM 14 days

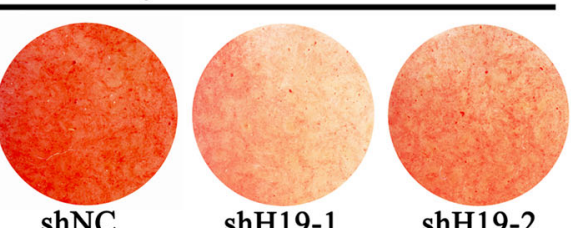

H19
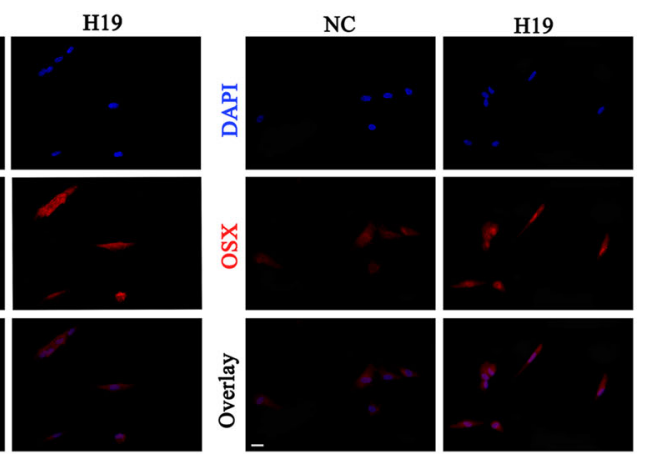

Fig. 2 (See legend on next page.) 
(see figure on previous page)

Fig. 2 LncRNA-H19 promotes osteo/odontogenesis of SCAPs. a Western blot results revealed that the protein levels of OCN, OSX, RUNX2, ALP and DSP significantly increased in $\mathrm{H} 19$ group while decreased in shH19-1 and shH19-2 groups after osteoblast induction for 7 days. GAPDH served as an internal control. Histograms showed the quantification of band intensities ( $P<0.05$, ${ }^{*} P<0.01$ ). b Relative mRNA expressions of OCN, OSX, RUNX2, ALP and DSPP measured by qRT-PCR after OB induction for 7 days. GAPDH was used for normalization. Results were presented as the mean \pm SD $\left({ }^{*} P<\right.$ 0.05 , ${ }^{* *} P<0.01$ ). $c$ Images of ALP staining in the NC, H19, shNC, shH19-1, and shH19-2 groups. SCAPs were cultured in growth medium (GM) or osteogenic medium (OM) for 7 days. Histograms show the activity of ALP were increased by H19 overexpression and decreased by H19 knockdown after OB induction for 7 days (**P<0.01). d After cell culture in GM or OM for 14 days, alizarin red staining showed that H19 group generated more calcified nodules than control group. shNC group generated more calcified nodules than shH19-1 and shH19-2 groups. e Histograms showed quantification of Alizarin red staining by spectrophotometry. Values were expressed as means $\pm S D$, ${ }^{*} P<0.01$. $\mathbf{f} I$ mmunofluorescence assay revealed that the expressions of DSP and OSX in Lenti-H19 treated SCAPs were significantly up-regulated (Scale Bar $=50 \mu \mathrm{m}$ )

(also called C-jun-amino-terminal kinase-interacting protein 4, JIP4) is a scaffold protein that is important in the activation of p38 and JNK pathways, which are closely related to the osteo/odontogenic differentiation of SCAPs $^{15,27}$. RIP assay demonstrated higher miR-141 expression $\mathrm{n}$ the anti-SPAG9 group compared with the anti-normal IgG group (Fig. 6a). As shown in Fig. 5b, qRT-PCR results revealed that SPAG9 level significantly decreased by miR-141 mimics and increased by miR-141 inhibitor $(P<0.05$ or $P<0.01)$. Western blot also proved that miR-141 suppressed protein expression of SPAG9 in SCAPs $(P<0.01$, Fig. $6 \mathrm{c})$. The above findings confirmed SPAG9 is a direct target of miR-141.

\section{SPAG9 activates p38 and JNK pathways in SCAPs}

To determine whether SPAG9 could activate p38 and JNK pathways which exert a crucial role in the committed differentiation of SCAPs, relative proteins in SCAPs treated with si-SPAG9 at $72 \mathrm{~h}$ were investigated. Phosphorylated level of p38 in cytoplasm did not change in siSPAG9 group as compared with si-NC group (Fig. 7a, b). However, the protein expression of phosphorylated JNK was obviously down-regulated in si-SPAG9 group compared with that in si-NC group. Co-treatment of the specific MAPK activator (anisomycin) reduced p-JNK/ JNK in si-SPAG9 + anisomycin group compared with anisomycin treated SCAPs. Moreover, p-p38/p38 also remarkably decreased in si-SPAG9 + anisomycin group compared with anisomycin group (Fig. 7a, b). The above findings indicated that SPAG9 could activate p38 and JNK pathways.

\section{MiR-141 inhibitor can rescue the downregulated effects caused by $\mathrm{H} 19$ deficiency}

To further investigate how miR-141 and SPAG9 were involved in H19-mediated differentiation, the rescure assays were performed. QRT-PCR results suggested that co-transfection with miR-141 inhibitor and shH19-1 significantly rescued SPAG9 expression in comparison to shH19-1 group (Fig. 7c). As presented in Fig. 7d, H19 deficiency-induced suppressed SPAG9, RUNX2, ALP, and DSP levels could be rescued in co-transfected cells $(P<$
0.05 or $P<0.01$, Fig. 7 e). In a word, lncRNA-H19 absorbs miR-141 as a ceRNA, increases the transcription of SPAG9, and activates the MAPK pathway to promote the committed differentiation of SCAPs (Fig. 7f).

\section{Discussion}

In recent years, lncRNAs have received widespread attention as emerging regulators with diverse biological functions. LncRNAs are transcripts with over 200 nucleotides and take part in various cellular performances including cell growth, pluripotency and differentiation ${ }^{28}$. LncRNA-H19 locates near the telomeric region of chromosome $11 \mathrm{p} 15.5$ and is maternally imprinted ${ }^{29}$. It is abundantly expressed during fetal life, and is abundantly expressed and conserved non-coding transcripts in mammalian development. LncRNA-H19 participates in the osteogenic differentiation of $\mathrm{BMSCs}^{26}$. However, the underlying mechanism in osteogenesis regulation has not been completely studied.

We demonstrated how H19 served as an osteo/odontogesis-related lncRNA in SCAPs. H19 expression was upregulated during the osteogenic differentiation of SCAPs, indicating that H19 may provoke the osteogenic differentiation of SCAPs. Some researchers have shown that $\mathrm{H} 19$ has a positive influence on the proliferation of $\mathrm{MSCs}^{30}$. We did not observe the regulatory effect of H19 on SCAPs proliferation between H19 group and control group, which may be explained by the different cell lineages. Moreover, previous studies reported that H19 can plays an inhibitive role in cell proliferation, suggesting the effect of lncRNA-H19 on regulation of cell proliferation is complicated ${ }^{31,32}$.

Rescue experiments were conducted to explore the regulatory effect of lncRNA-H19 on the osteogenic differentiation of SCAPs. We found the osteo/odontogenic markers (e.g., ALP, RUNX2) were significantly upregulated in H19 overexpression group, which were downregulated after H19 knockdown. RUNX2 is part of the RUNX gene family, which has a primary function in osteoblast differentiation and directly stimulates the transcription of osteoblast-related gene (e.g., OSX and $\mathrm{ALP})^{33}$. OCN is primarily generated by osteoblasts in the 
A

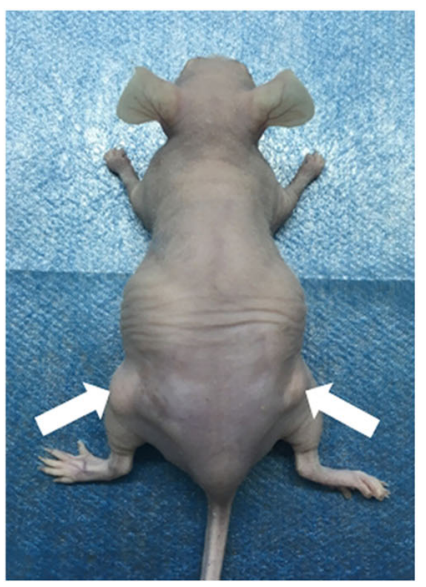

$\mathrm{C}$
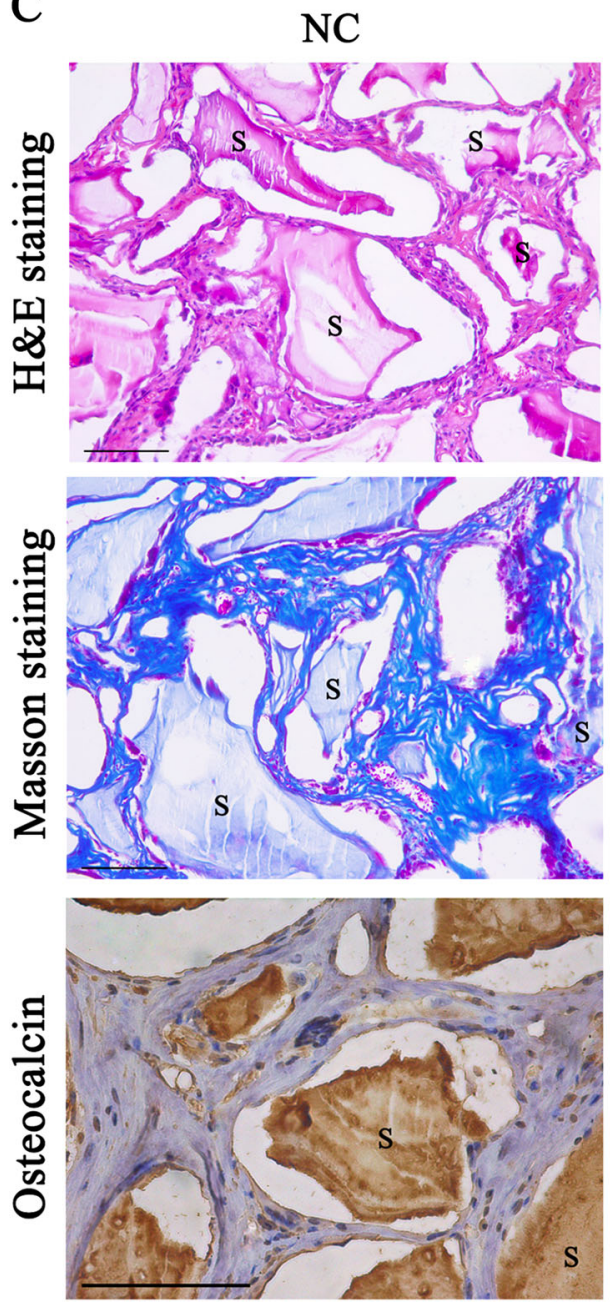

B

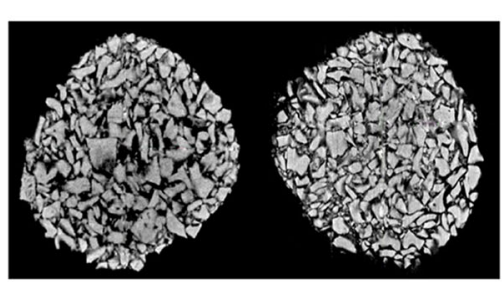

$\mathrm{NC}$

H19

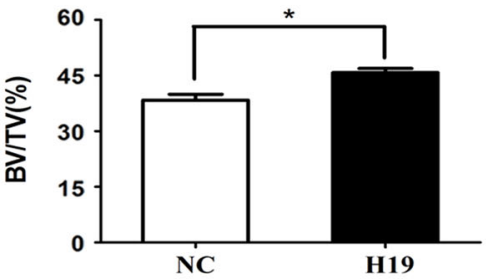

H19
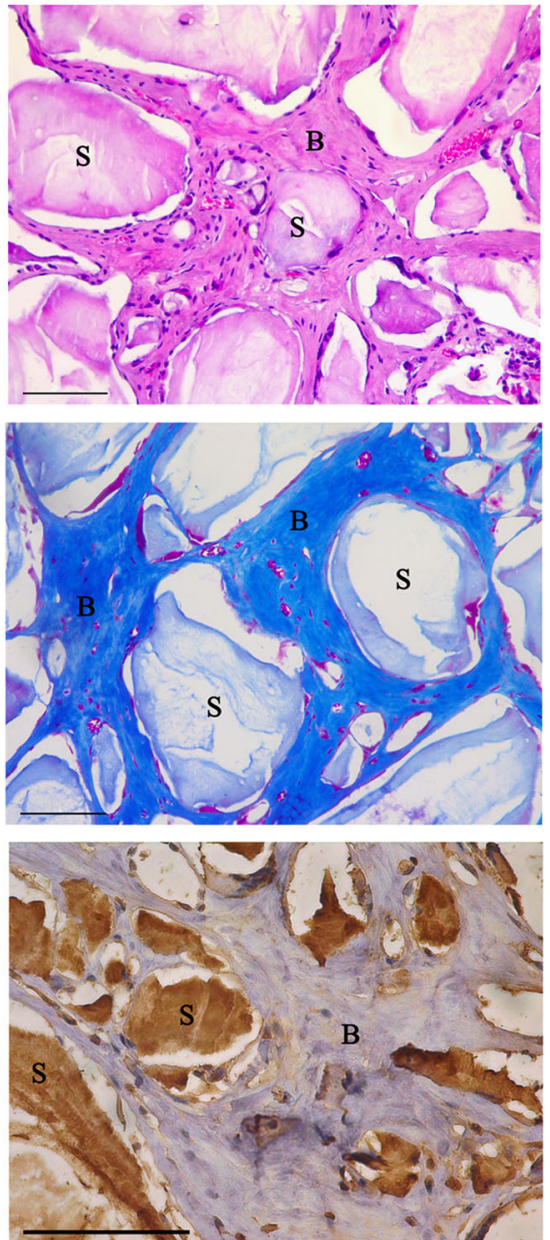

Fig. $3 \mathrm{H} 19$ enhanced the osteo/dentinogenesis of SCAPs in vivo. a SCAPs in NC and H19 group were transplanted subcutaneously into 5-weekold BALB/C homozygous nude mice for 8 weeks. $\mathbf{b}$ Upper: reconstructed three-dimensional micro-CT images of the tissue-engineered bone constructs from NC and H19 groups. Lower: percentages of new BV/TV of cultured bone constructs. Data are shown as the mean \pm SD $\left({ }^{*} P<0.05\right)$. c H\&E staining, Masson staining and immunohistochemical staining of osteocalcin in NC and H19 groups. B bone/dentin-like tissues, $\mathrm{S}$ around the scaffold, BV/TV bone volume to tissue volume, NC negative control. Scale Bar $=100 \mu \mathrm{m}$ 


\section{A Predicted consequential pairing of target region and miRNA}

H19 5' C

$\operatorname{miR-141} 3{ }^{\prime}$

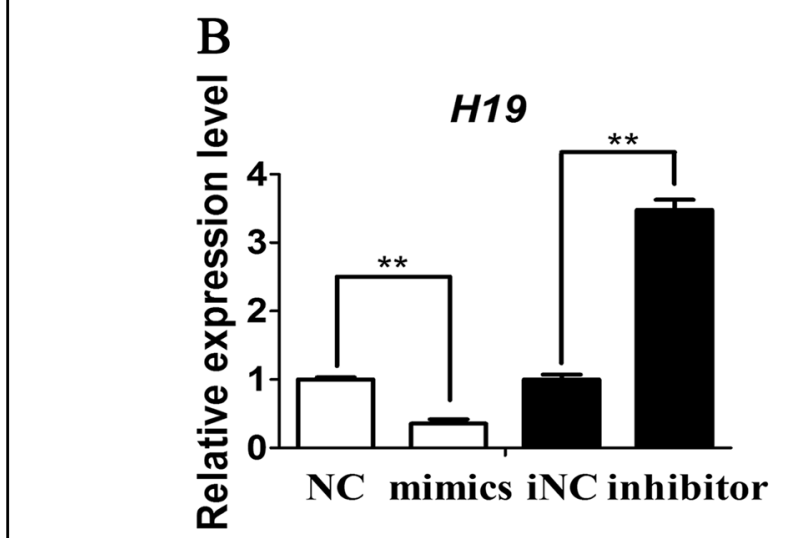

AA

$\mathrm{C}$

\author{
GU<smiles></smiles>

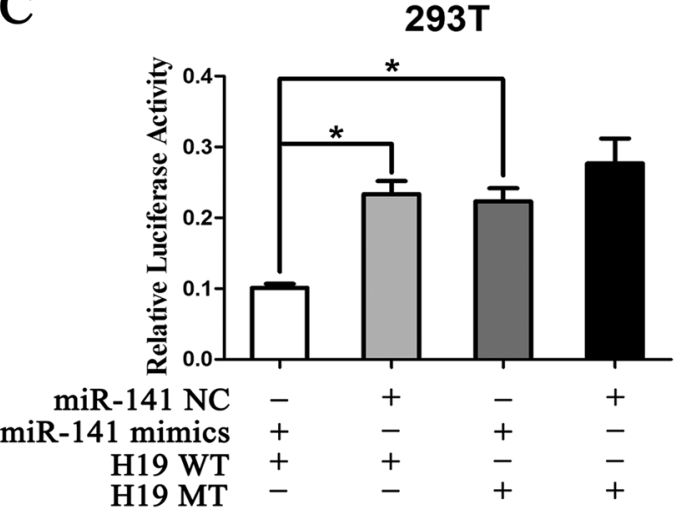

Fig. 4 LncRNA-H19 functions as an endogenous sponge of miR-141. a The potential binding sites between IncRNA-H19 and miR-141 predicted by biological software. $\mathbf{b}$ Relative IncRNA-H19 expression level in SCAPs transfected with with miR-141 mimics or miR-141 inhibitor (**P<0.01). c Luciferase reporter assay was used to validate the target in 293T cells. The relative luciferase activities of luciferase reporters containing WT or Mut IncRNA-H19 were assayed $48 \mathrm{~h}$ after co-transfection with miR-141 mimics or mimics NC. Relative Renilla luciferase activity was normalized to that of firefly luciferase $\left(* P<0.05,{ }^{*} P<0.01\right)$

late stage of osteoblastic differentiation, and therefore its serum concentration could indicate bone formation ${ }^{34}$. As a specific marker of odontoblast, DSPP is mostly present in the secretory odontoblasts and DSP shows great influences in odontoblast differentiation and dentin mineralization ${ }^{35}$. In addition, ALP staining, alizarin red staining, and immunofluorescence staining assays further confirmed that H19 strongly promotes the osteo/odontogenic differentiation of SCAPs. Moreover, the in vivo study demonstrated that H19-overexpressing SCAPs displayed enhanced bone formation capacity.

Although $\mathrm{H} 19$ was demonstrated to be involved in the osteo/odontogenesis of SCAPs, the exact molecular mechanism remains to be elucidated. Recently, the "ceRNA" hypothesis is commonly accepted that lncRNA acquires functionality by acting as sponge of microRNA and abolishes microRNA's inhibitory action to target mRNAs if there is a binding site between lncRNA and microRNA ${ }^{21}$. For instance, recent findings have demonstrated that $\mathrm{H} 19$ absorbs and antagonizes microRNAs in the let-7 family, resulting in the downregulated proteincoding genes targeted by let- $7^{36}$. In addition, H19 regulates mechanical tension-induced osteogenesis of BMSCs by absorbing miR-138 and up-regulates its downstream FAK ${ }^{37}$. LncRNA-H19 can enhance MSCs survival and angiogenic capacity by acting as a molecular sponge for miR-199a, eventually regulating in vitro expression of VEGFA ${ }^{30}$. To explore the underlying prooncogenic mechanism of $\mathrm{H} 19$ in this study, bioinformatics analysis indicated that miR-141 can bind to lncRNA-H19. MiR-141 belongs to the same cluster as the miR-200 family and has been observed to negatively regulate cellular senescence by inhibiting ZMPSTE24 expression ${ }^{38}$. However, the functions and potential targets of miR-141 in SCAPs remain to be fully elucidated. Thus, the effect of miR-141 on osteo/odontogenic differentiation of SCAPs and the relation between miR-141 and lncRNA-H19 were further explored. Firstly, our results verified that miR-141 negatively regulates osteo/odontogenic differentiation of SCAPs: miR-141 overexpression down-regulated mineral-related proteins/genes (OCN/ OCN, OSX/OSX, RUNX2/RUNX2, ALP/ALP, DSP/DSPP) and matrix mineralization, whereas the miR-141 inhibitor up-regulated all of the mineral-related proteins/genes and matrix mineralization. Secondly, miR-141 expression was negatively regulated by H19: overexpression of H19 decreased miR-141 while H19 inhibition elevated miR141 expression. To ascertain if there is direct binding between IncRNA-H19 and miR-141, luciferase reporter gene assay was conducted. MiR-141 inhibited the luciferase activity of vector containing H19 sequence. Mutation of the putative miR-141 target sites reversed the 


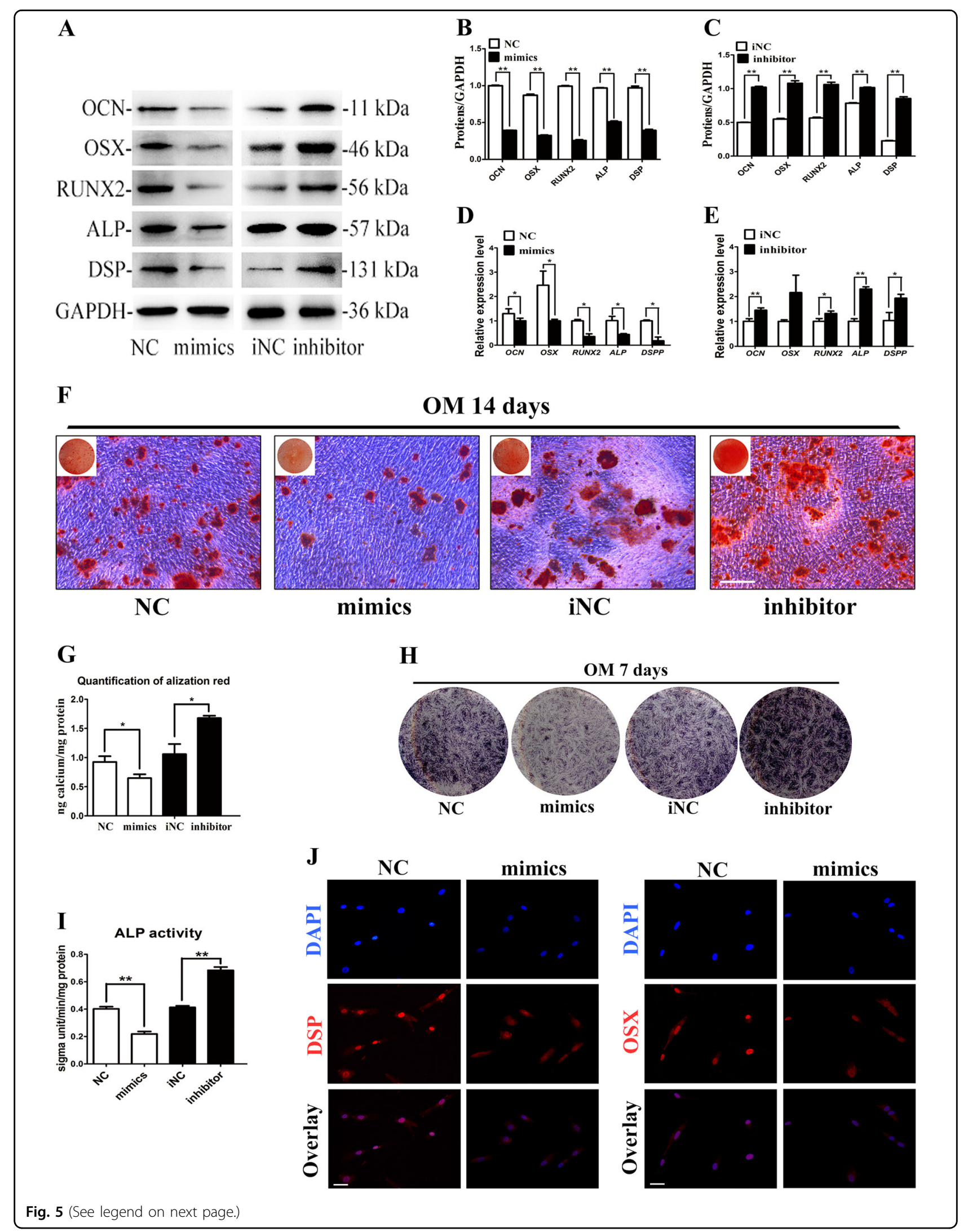


(see figure on previous page)

Fig. 5 MiR-141 inhibits osteo/odontogenic differentiation of SCAPs. a-c Western blot results revealed that the protein levels of OCN, OSX, RUNX2, ALP and DSP significantly decreased in miR-141 mimics group while increased in miR-141 inhibitor group (**P<0.01). d, e Relative mRNA expressions of OCN, OSX, RUNX2, ALP, and DSPP were measured by qRT-PCR. GAPDH was used for normalization. Results were presented as the mean $\pm \mathrm{SD}\left({ }^{*} P<0.05 ;{ }^{*} P<0.01\right)$. f, $\mathbf{g}$ After cell culturing in OM for 14 days, alizarin red staining showed that miR-141 mimics group generated more calcified nodules than control group. MiR-141 inhibitor group generated less calcified nodules than iNC group (Scale Bar $=200 \mu \mathrm{m}$ ). Histograms showed quantification of Alizarin red staining by spectrophotometry. Values were expressed as means $\pm S D,{ }^{* *} P<0.01$. $\mathbf{h}$, $\mathbf{i}$ Images of ALP staining in the NC, mimics, iNC and inhibitor groups. SCAPs were cultured in OM for 7 days. Histograms showed the activity of ALP increased by miR-141 overexpression and decreased by miR-141 knockdown (**P<0.01). $\mathbf{j}$ Immunofluorescence assay revealed that the expressions of DSP and OSX were significantly up-regulated in miR-141 mimics group compared with NC group (Scale Bar $=50 \mu \mathrm{m}$ )
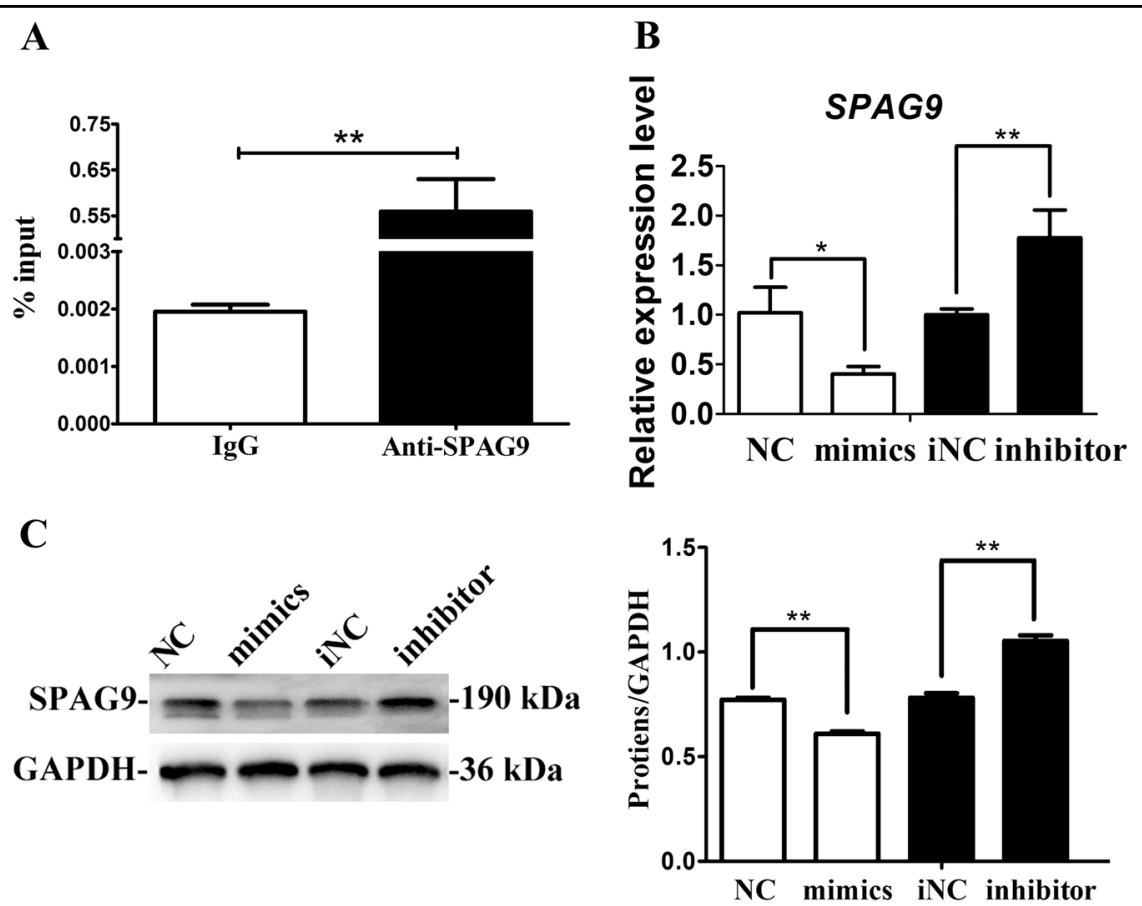

Fig. 6 MiR-141 downregulates the expression of SPAG9. a Relative expression level of miR-141 in SCAPs was accessed by RIP assay $(* * P<0.01)$. b Relative mRNA expression of SPAG9 in SCAPs transfected with with miR-141 mimics or miR-141 inhibitor $\left({ }^{*} P<0.05,{ }^{*} P<0.01\right)$. c Western blot analysis of protein expression of SPAG9 in SCAPs transfected with miR-141 mimics or inhibitor. $\mathbf{d}$ Results of western blotting was analyzed with ImageJ software and data were presented as ratio of target protein to GAPDH (**P<0.01)

previous suppressive effect, indicating that lncRNA-H19 directly binds to miR-141 via the putative MRE in this "ceRNA" regulatory network.

MAPK signaling pathway exerts a vital role in mammals' cellular regulations including apoptosis, proliferation, and differentiation ${ }^{39}$. C-Jun N-terminal kinase (JNK), extracellular signal-regulated kinase (ERK) and p38 MAPK are the major components of MAPK pathway ${ }^{40}$. Indeed, our previous studies have identified that MAPK pathway participates in osteo/odontogenic differentiation of tooth-derived MSCs ${ }^{41-44}$. It is reported that the $3^{\prime} \mathrm{UTR}$ of SPAG9 gene contains a sequence complementary to the miR-141 seed region, revealing miR-141 can directly target SPAG9 ${ }^{23}$. Interestingly, the protein encoded by SPAG9 gene, also known as JIP4, is an important scaffold protein of MAPK pathway ${ }^{27}$. It activates MAPK pathway by regulating p38 and JNK phosphorlyation ${ }^{45,46}$. Hence, we hypothesized that IncRNA-H19 up-regulates SPAG9 by sponging miR-141, thus mediating osteo/odontogenic differentiation of SCAPs through MAPK pathway. To validate our hypothesis, RIP assays was performed at first. Results revealed that miR-141 could epigenetically inhibited the expressions of SPAG9 (Fig. 6a). Moreover, SCAPs transfected with miR-141 mimics downregulated the SPAG9 expression while miR-141 inhibitor transfection upregulated its expression (Fig. 6b, c). Collectively, all these data indicated that miR-141 targets the 3'UTR of the SPAG9 gene, suppressing mRNA translation of SPAG9 at the post-transcriptional level. Secondly, the interaction between MAPK pathway and SPAG9 was 
A

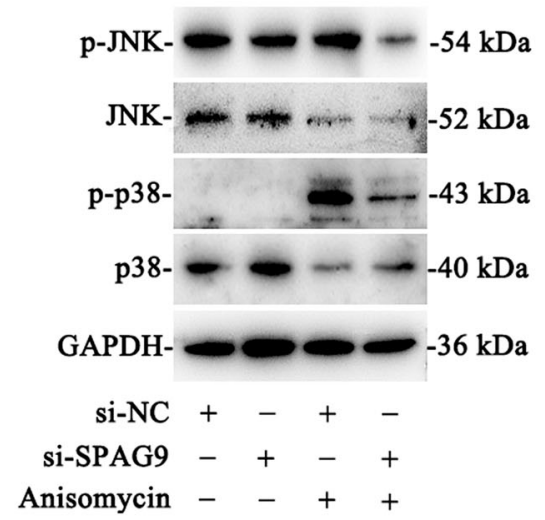

C

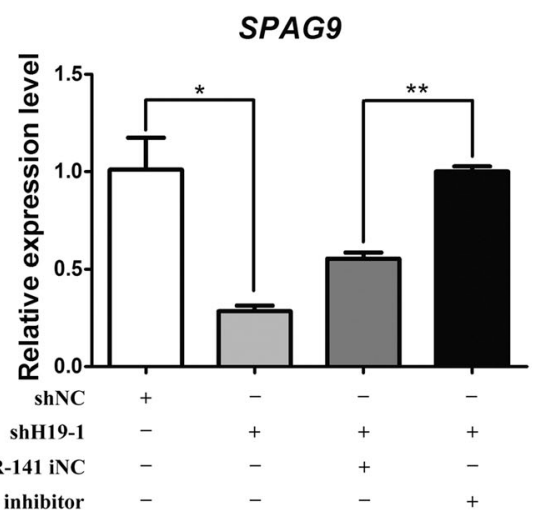

$\mathbf{E}$

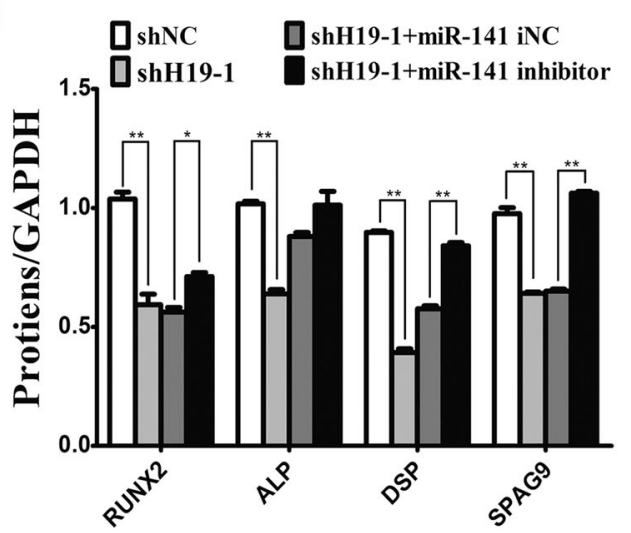

B

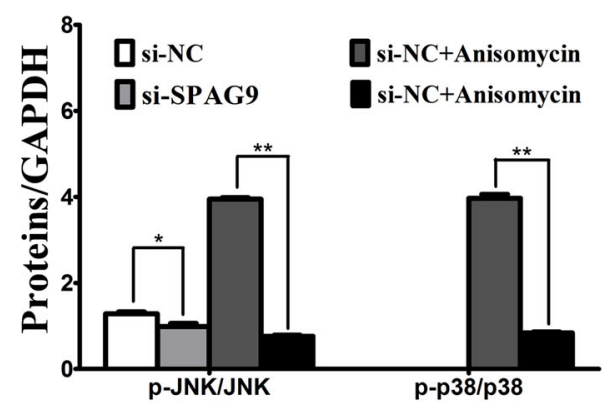

D

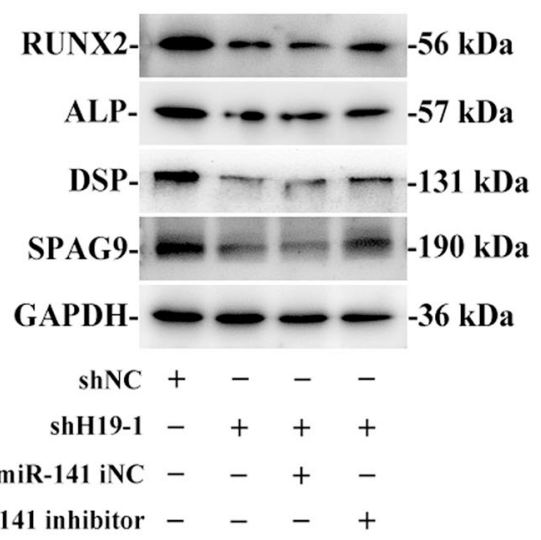

\section{F}

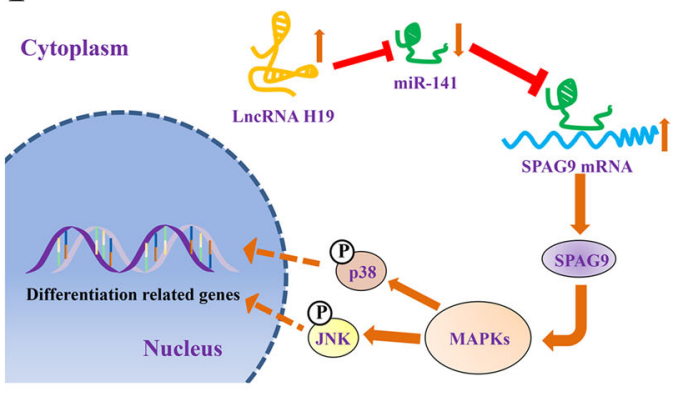

Fig. 7 SPAG9 regulates $\mathrm{p} 38$ and JNK signaling pathways in SCAPs and the miR-141 inhibitor could rescue the shH19-1 mediated inhibitory effects of osteo/odontogenic differentiation in SCAPs. a Western blot assay for the expressions of genes relative to p38 and JNK signaling pathways at $72 \mathrm{~h}$. $\mathbf{b}$ The ratio changes of $\mathrm{p}-\mathrm{p} 38 / \mathrm{p} 38$ and $\mathrm{p}-\mathrm{JNK} / \mathrm{JNK}$ at $72 \mathrm{~h}$ in different groups. Values were described as the means $\pm S D, n=3$. ${ }^{*} P<0.05,{ }^{* *} P<0.01$. c Results of qRT-PCR analysis revealed the miR-141 inhibitor rescued the shH19-1 mediated downregulation of SPAG9 expression $\left({ }^{*} P<0.05,{ }^{* *} P<0.01\right)$. d Results of western blot analysis indicated that the miR-141 inhibitor rescued the shH19-1 mediated downregulation of RUNX2, ALP, DSP, SPAG9. e Results of western blotting was analyzed with ImageJ software and data were presented as ratio of target protein to GAPDH in the form of grayscale value. $\left({ }^{*} P<0.05,{ }^{*} P<0.01\right)$. f Schematic diagram for IncRNA-H19/miR-141/SPAG9/MAPK axis

evaluated. As we expected, the protein expression of $\mathrm{p}$ JNK was reduced by SPAG9 knockdown. Co-treatment with the specific MAPK activator (anisomycin) downregulated $\mathrm{p}-\mathrm{JNK} / \mathrm{JNK}$ and $\mathrm{p}-\mathrm{p}-38 / \mathrm{p}-38$ in si-SPAG9 + anisomycin group compared with anisomycin treated
SCAPs, indicating that SPAG9 could activate the p-38 and JNK signaling pathways in SCAPs. To further validate whether lncRNA-H19 regulated SPAG9 expression by sponging miR-141 in osteo/odontogenic differentiation of SCAPs, we performed the rescue assays. Co-transfection 
of miR-141 inhibitor with shH19-1 significantly rescued SPAG9 mRNA compared with the sh-H19 groups (Fig. 7c). Besides, the downregulation of miR-141 reversed the inhibitory effects of shH19-1 on the protein expressions of SPAG9 and other genes relative to osteo/odontogenic differentiation (Fig. 7d, e). Together, these results indicated that IncRNA-H19 epigenetically promoted SPAG9 transcription by interacting with miR-141.

To sum up, this study elucidated the osteogenic function of lncRNA-H19 in SCAPs, in which "IncRNA-H19/ miR-141/SPAG9/MAPK" positive feedback loop plays a paramount role. Our results may provide references for revealing molecular mechanism of the odonto/osteogenic differentiation of SCAPs, and therapeutic targets in the future. However, whether other feedback loops take part in this ceRNA regulation are still needed to be further elucidated.

\section{Acknowledgements}

This work was supported by National Natural Science Foundation of China (81600822, 81873707), Medical Talent Project of Jiangsu Province (ZDRCA2016086), the Priority Academic Program Development of Jiangsu Higher Education Institutions (PAPD, 2018-87) and Science and Technology Development Project of Jiangsu Province (BE2017731).

\section{Author contributions}

Z.L., M.Y., Y.Y., Y.W. and G.L. performed the experiments and analyzed the data Z.L., M.Y. and J.Y. designed the study. Z.L., Y.P., N.L., R.G. and J.Y. wrote the manuscript. All authors reviewed and revised the manuscript.

\section{Conflict of interest}

The authors declare that they have no conflict of interest.

\section{Publisher's note}

Springer Nature remains neutral with regard to jurisdictional claims in published maps and institutional affiliations.

Supplementary Information accompanies this paper at (https://doi.org/ 10.1038/s41419-019-1337-3).

Received: 23 October 2018 Revised: 10 December 2018 Accepted: 2 January 2019

Published online: 12 February 2019

\section{References}

1. Bianco, P. et al. The meaning, the sense and the significance: translating the science of mesenchymal stem cells into medicine. Nat. Med. 19, 35-42 (2013).

2. Kim, H. J. et al. Transfection of gene regulation nanoparticles complexed with pDNA and shRNA controls multilineage differentiation of hMSCs. Biomaterials 177, 1-13 (2018).

3. Chen, Q. et al. Fate decision of mesenchymal stem cells: adipocytes or osteoblasts? Cell Death Differ. 23, 1128-1139 (2016).

4. LV, F. J., Tuan, R. S., Cheung, K. M. \& Leung, V. Y. Concise review: the surface markers and identity of human mesenchymal stem cells. Stem Cells $\mathbf{3 2}$ 1408-1419 (2014).

5. Zhang, C. et al. Therapeutic effect of dental pulp stem cell transplantation on a rat model of radioactivity-induced esophageal injury. Cell Death Dis. 9, 738 (2018).

6. Shi, S. \& Gronthos, S. Perivascular niche of postnatal mesenchymal stem cells in human bone marrow and dental pulp. J. Bone Miner. Res 18, 696-704 (2003).
7. Sonoyama, W. et al. Characterization of the apical papilla and its residing stem cells from human immature permanent teeth: a pilot study. J. Endod. 34, 166-171 (2008).

8. Liu, J. et al. Concise reviews: characteristics and potential applications of human dental tissue-derived mesenchymal stem cells. Stem Cells 33, 627-638 (2015).

9. Yang, C., Li, X., Sun, L., Guo, W. \& Tian, W. Potential of human dental stem cells in repairing the complete transection of rat spinal cord. J. Neural Eng. 14, 026005 (2017).

10. Sonoyama, W. et al. Mesenchymal stem cell-mediated functional tooth regeneration in swine. PLOS ONE 1, e79 (2006).

11. Zhang, H. et al. Canonical Wnt signaling acts synergistically on BMP9-induced osteo/odontoblastic differentiation of stem cells of dental apical papilla (SCAPs). Biomaterials 39, 145-154 (2015).

12. Wang, Y. et al. MicroRNA hsa-let-7b suppresses the odonto/osteogenic differentiation capacity of stem cells from apical papilla by targeting MMP1. J. Cell Biochem 119, 6545-6554 (2018).

13. LV, T. et al. Insulin-like growth factor 1 promotes the proliferation and committed differentiation of human dental pulp stem cells through MAPK pathways. Arch. Oral. Biol. 72, 116-123 (2016).

14. Yan, M. et al. Mineral trioxide aggregate promotes the odonto/osteogenic differentiation and dentinogenesis of stem cells from apical papilla via nuclear factor kappa B signaling pathway. J. Endod. 40, 640-647 (2014).

15. Li, Y. et al. 17beta-estradiol promotes the odonto/osteogenic differentiation of stem cells from apical papilla via mitogen-activated protein kinase pathway. Stem Cell Res. Ther. 5, 125 (2014).

16. Tye, C. E. et al. Could IncRNAs be the missing links in control of mesenchymal stem cell differentiation? J. Cell Physiol. 230, 526-534 (2015).

17. Lu, L., Dai, Z., Luo, Q. \& Lv, G. The long noncoding RNA cancer susceptibility candidate 2 inhibits tumor progression in osteosarcoma. Mol. Med Rep. 17 1947-1953 (2018).

18. He, Q. et al. Long noncoding RNA TUG1 facilitates osteogenic differentiation of periodontal ligament stem cells via interacting with Lin28A. Cell Death Dis. 9, 455 (2018).

19. Zhuang, W. et al. Upregulation of IncRNA MEG3 promotes osteogenic differentiation of mesenchymal stem cells from multiple myeloma patients by targeting BMP4 transcription. Stem Cells 33, 1985-1997 (2015).

20. Zhu, L. \& Xu, P. C. Downregulated LncRNA-ANCR promotes osteoblast differentiation by targeting EZH2 and regulating Runx2 expression. Biochem. Biophys. Res Commun. 432, 612-617 (2013).

21. Yuan, N. et al. Integrative analysis of InCRNAs and miRNAs with coding RNAs associated with ceRNA crosstalk network in triple negative breast cancer. Onco. Targets Ther. 10, 5883-5897 (2017).

22. Shuwen, H., Qing, Z., Yan, Z. \& Xi, Y. Competitive endogenous RNA in colorectal cancer: a systematic review. Gene 645, 157-162 (2017).

23. Lou, G. et al. Direct targeting sperm-associated antigen 9 by miR-141 influences hepatocellular carcinoma cell growth and metastasis via JNK pathway. J. Exp. Clin. Cancer Res. 35, 14 (2016).

24. Weng, R. et al. Long noncoding RNA-1604 orchestrates neural differentiation through the miR-200c/ZEB axis. Stem Cells 36, 325-336 (2017).

25. Zhang, L. et al. TUG1 knockdown ameliorates atherosclerosis via up-regulating the expression of miR-133a target gene FGF1. Cardiovasc. Pathol. 33, 6-15 (2017).

26. Huang, Y., Zheng, Y., Jia, L., Li, W. \& Long Noncoding, R. N. A. H19 promotes osteoblast differentiation via TGF-beta1/Smad3/HDAC signaling pathway by deriving miR-675. Stem Cells 33, 3481-3492 (2015).

27. Tanaka, T., lino, M. \& Goto, K. Knockdown of Sec8 enhances the binding affinity of c-Jun N-terminal kinase (JNK)-interacting protein 4 for mitogenactivated protein kinase kinase 4 (MKK4) and suppresses the phosphorylation of MKK4, p38, and JNK, thereby inhibiting apoptosis. FEBS J. 281, 5237-5250 (2014).

28. Liang, Y. et al. A novel long non-coding RNA-PRLB acts as a tumor promoter through regulating miR-4766-5p/SIRT1 axis in breast cancer. Cell Death Dis. 9, 563 (2018).

29. Peng, F. et al. H19/let-7/LIN28 reciprocal negative regulatory circuit promotes breast cancer stem cell maintenance. Cell Death Dis. 8, e2569 (2017).

30. Hou, J. et al. Long noncoding RNA H19 upregulates vascular endothelial growth factor A to enhance mesenchymal stem cells survival and angiogenic capacity by inhibiting miR-199a-5p. Stem Cell Res. Ther. 9, 109 (2018).

31. Wang, S. et al. Long noncoding RNA H19 inhibits the proliferation of fetal liver cells and the Wnt signaling pathway. FEBS Lett. 590, 559-570 (2016). 
32. Han, Y. et al. Downregulation of long non-coding RNA H19 promotes P19CL6 cells proliferation and inhibits apoptosis during late-stage cardiac differentiation via miR-19b-modulated Sox6. Cell Biosci. 6, 58 (2016).

33. Cohen, M. M. Jr. Perspectives on RUNX genes: an update. Am. J. Med. Genet. A 149A, 2629-2646 (2009)

34. Mizokami, A., Kawakubo-Yasukochi, T. \& Hirata, M. Osteocalcin and its endocrine functions. Biochem. Pharmacol. 132, 1-8 (2017).

35. lejima, D., Sumita, Y., Kagami, H., Ando, Y. \& Ueda, M. CC-chemokine family protein MIP-3alpha in human mesenchymal stem cells. Arch. Oral. Biol. 52, 924-931 (2007).

36. Kallen, A. N. et al. The imprinted H19 IncRNA antagonizes let-7 microRNAs. Mol. Cell 52, 101-112 (2013).

37. $\mathrm{Wu}$, J. et al. Long non-coding RNA H19 mediates mechanical tension-induced osteogenesis of bone marrow mesenchymal stem cells via FAK by sponging miR-138. Bone 108, 62-70 (2018).

38. Yu, K. R. et al. MicroRNA-141-3p plays a role in human mesenchymal stem cell aging by directly targeting ZMPSTE24. J. Cell Sci. 126(Pt 23), 5422-5431 (2013).

39. Raman, M., Chen, W. \& Cobb, M. H. Differential regulation and properties of MAPKs. Oncogene 26, 3100-3112 (2007).
40. Sumbayev, V. V. \& Yasinska, I. M. Regulation of MAP kinase-dependent apoptotic pathway: implication of reactive oxygen and nitrogen species. Arch. Biochem Biophys. 436, 406-412 (2005).

41. Liu, G. X. et al. Hsa-let-7c controls the committed differentiation of IGF-1treated mesenchymal stem cells derived from dental pulps by targeting IGF$1 \mathrm{R}$ via the MAPK pathways. Exp. Mol. Med. 50, 25 (2018).

42. Wang, Y. et al. Mineral trioxide aggregate enhances the osteogenic capacity of periodontal ligament stem cells via NF-kappaB and MAPK signaling pathways. J. Cell Physiol. 233, 2386-2397 (2018).

43. Wang, Y., Li, J., Song, W. \& Yu, J. Mineral trioxide aggregate upregulates odonto/osteogenic capacity of bone marrow stromal cells from craniofacial bones via JNK and ERK MAPK signalling pathways. Cell Prolif. 47, 241-248 (2014).

44. Yu, Y. et al. Dentin matrix proteins (DMPs) enhance differentiation of BMMSCs via ERK and P38 MAPK pathways. Cell Tissue Res. 356, 171-182 (2014).

45. Li, R. et al. JLP-JNK signaling protects cancer cells from reactive oxygen species-induced cell death. Biochem. Biophys. Res. Commun. 501, 724-730 (2018).

46. Whitmarsh, A. J. The JIP family of MAPK scaffold proteins. Biochem. Soc. Trans. 34(Pt 5), 828-832 (2006) 